\title{
Vers une valorisation du savoir-faire local des oasis : cas du jus de dattes Tassabount au Maroc
}

\author{
Hasnaâ HARRAK $^{1 *}$, Marc Lebrun ${ }^{2}$, Moulay Mustapha IsmaïLI AlaOuI ${ }^{3}$, Ahmed Faouzi SenHAJI $^{3}$, Allal HAMOUda $^{3}$
}

${ }^{1}$ Inst. Ntl. Rech. Agron., Cent. Rég. Rech. Agron. Marrakech, INRA, BP 533,

Marrakech 40000 ,

Maroc

h.harrak@yahoo.fr

2 CIRAD, Persyst,

UMR Qualisud,

34398 Montpellier Cedex 5,

France

${ }^{3}$ Inst. Agron. Vét. Hassan II, BP 6202-Instituts,

Rabat 10 101,

Maroc

\section{Towards a valorization of local oasis know-how: the case of Tassabount date juice in Morocco.}

Abstract - Introduction. The Moroccan oases are well known for the ingenious know-how of their inhabitants in the management and the preservation of natural resources. The date, the main product of the oases, benefits greatly from this ingeniousness, which nevertheless nowadays is less exploited and risks being forgotten. This study aimed at documenting and analyzing the know-how of date juice processing, locally called Tassabount, and at proposing ways of improving it. Materials and methods. The methodology adopted for the documenting work and the analysis of the local knowledge of date juice processing was based on a participatory approach involving one hundred oasis women from Drâa valley (Morocco). Results and discussion. Our study highlighted an ingenious practice in local date processing into juice. This know-how, developed by women living in the oases, is closely related to the genetic diversity of date palm (varieties and hybrids). In addition, the Tassabount is identified as a promising product to valorize, by virtue of its organoleptic and therapeutic qualities. Furthermore, description and analysis of the familial Tassabount process helped find ways to improve it, to be ready to transfer to traditional and/or industrial production.

Morocco / Pbonix dactylifera / dates / drug plants / culinary herbs / fruit juices / oases / traditional technology / modernization / appropriate technology / technology transfer

\section{Vers une valorisation du savoir-faire local des oasis : cas du jus de dattes Tassabount au Maroc.}

Résumé - Introduction. Les oasis marocaines sont réputées pour le savoir-faire ingénieux de leurs habitants en matière de gestion et de préservation des ressources naturelles. La datte, principal produit des oasis, bénéficie amplement de cette ingéniosité qui connaît, de nos jours, une régression et un manque de valorisation. Notre étude a eu pour objectif d'archiver et d'analyser le savoir-faire de la transformation des dattes en jus, localement appelé Tassabount, et d'en proposer un mode de valorisation. Matériel et méthodes. La méthodologie employée pour le travail d'archivage et d'analyse des connaissances locales de transformation

* Correspondance et tirés à part des dattes en jus a été basé sur une approche participative impliquant une centaine de femmes oasiennes de la vallée du Drâa (Maroc). Résultats et discussion. Notre étude a mis en évidence une pratique ingénieuse dans la transformation locale des dattes en jus. Ce savoirfaire, développé par les femmes oasiennes, est étroitement lié à la diversité génétique du palmier dattier (variétés et hybrides). En outre, le Tassabount est identifié comme produit prometteur à valoriser, en misant sur ses qualités organoleptiques et ses vertus thérapeutiques. Par ailleurs, la description et l'analyse du procédé familial de préparation du Tassabount ont permis d'identifier des voies possibles d'amélioration pour son élaboration et sa stabilisation en vue de son transfert à des entreprises artisanales et/ou industrielles.

Fruits, 2009, vol. 64, p. 253-2 (C) 2009 Cirad/EDP Sciences All rights reserved DOI: $10.1051 /$ fruits/2009019 www.fruits-journal.org

RESUMEN EsPAÑoL, p. 260
Maroc / Pboenix dactylifera / datte / plante médicinale / herbe aromatique / jus de fruits / oasis / technologie traditionnelle / modernisation / technologie appropriée / transfert de technologie 


\section{Introduction}

Les oasis marocaines ont un rôle stratégique dans les zones arides et leurs habitants sont réputés pour leur savoir-faire ingénieux en matière de gestion et de préservation des ressources locales. Parmi les ressources qui bénéficient amplement de cette ingéniosité, le palmier dattier (Phoenix dactylifera) constitue l'une des principales composantes de l'activité agricole des oasiens. Cela n'est pas dû uniquement à des facteurs environnementaux, religieux, culturels ou sociaux, mais aussi, et surtout, au fait que les dattes, fruits du palmier dattier, constituent une ressource alimentaire et médicinale importante pour la population oasienne [1]. L'ingéniosité liée à l'utilisation de la datte allie des caractéristiques portant sur l'aromatisation, la conservation et la médication en intégrant, dans les préparations à base de dattes, des plantes aromatiques et médicinales. Ainsi, les transformations locales sont importantes : d'une part, elles permettent de consommer les dattes de façon différée et de valoriser celles de moindre valeur marchande et de faible aptitude à la conservation; d'autre part, les plantes aromatiques et médicinales ajoutées à la quasi-totalité de ces préparations donnent au produit final une note aromatique et des vertus thérapeutiques très appréciées [1, 2].

Cependant, aujourd'hui, le savoir-faire local connaît une nette régression qui le menace de disparition. En effet, les personnes qui pratiquent et transmettent ce savoir-faire se raréfient et la valorisation des produits préparés localement est insuffisante [3].

Dans un tel contexte, les techniques de préparations locales à base de dattes utilisant le savoir-faire des femmes oasiennes mériteraient d'être archivées et la consommation de ces produits devrait être valorisée et promue par une insertion dans la cuisine marocaine et l'art culinaire moderne. En outre, les potentialités touristiques importantes des oasis marocaines, ajoutées à une diaspora oasienne vivant dans les différentes régions du Maroc et à l'étranger, militent en faveur d'un produit de terroir à base de dattes qui porterait un "label oasien". L'identité des produits issus de ce savoir- faire local serait apte à leur conférer une plus-value liée à leur authenticité, leurs caractéristiques particulières et leur qualité.

L'étude que nous avons effectuée propose d'apporter une contribution à la connaissance et à l'analyse du savoir-faire local de transformation des dattes en jus Tassabount. Dans la perspective de l'exploitation de marchés locaux et nationaux, elle vise également à décrire et à proposer des voies d'amélioration des pratiques familiales en vue de la mise au point et du transfert d'une technologie rénovée aux entreprises artisanales oasiennes, voire à des unités industrielles.

\section{Matériel et méthodes}

\subsection{Contexte lié au jus de dattes}

Dans le contexte actuel de la globalisation, les produits de terroir, très recherchés localement, pourraient également être commercialisés avec succès sur les marchés régionaux, nationaux et internationaux. Les produits à base de dattes, préparés dans les oasis marocaines, sont pour la plupart peu connus, même si certains peuvent cependant être valorisés en produits de terroir et émerger sur des marchés de niches. Le jus de dattes, connu localement sous l'appellation Tassabount, préparé avec des extraits de plantes aromatiques et médicinales, illustre bien cette situation.

Le Tassabount figurant parmi les préparations familiales à base de dattes a une qualité gustative indéniable et est très apprécié par la population oasienne. Il doit son expression aromatique et ses vertus organoleptiques et thérapeutiques aux variétés de dattes et aux plantes aromatiques et médicinales qui entrent dans sa préparation. Il possède en outre une signification spirituelle positive liée à son caractère mousseux. Au vu de cette situation particulièrement prometteuse, le Tassabount pourrait se positionner hors cadre familial pour émerger sur le marché à condition d'entreprendre des actions appropriées de valorisations artisanales et/ou industrielles. 
La fabrication de ce jus apparaît également essentielle pour pérenniser un procédé familial de préparation détenu uniquement par les femmes et hérité de pratiques ancestrales qui perdurent depuis de nombreuses générations ; par ailleurs, la préparation du Tassabount contribue à la valorisation des produits de terroir des oasis marocaines classées par l'UNESCO comme patrimoine mondial : "Réserve de biosphère des oasis du Sud marocain". Cette production permet aussi d'exploiter les dattes marocaines de faible valeur marchande à travers une activité créatrice de revenus et d'emplois, assurant un produit transformé de qualité.

\subsection{Collecte des informations}

La méthodologie adoptée dans notre étude pour la collecte des informations relatives à la préparation du Tassabount a été basée sur une approche participative impliquant une centaine de femmes oasiennes de la vallée du Drâa dans le Sud-est marocain. Cela s'est fait sans questionnaire d'enquête spécifique mais à l'aide de discussions / interviews menées avec le panel considéré en s'appuyant sur un guide d'entretien permettant de poser des questions préétablies.

\section{Résultats et discussion}

\subsection{Description et analyse du procédé familial de préparation du Tassabount}

Le jus mousseux et aromatisé à base de dattes et d'extrait aqueux des plantes aromatiques et médicinales, préparé dans les oasis marocaines, est appelé localement Tassabount, qui signifie "savon " en langue berbère, ou Mriss, qui désigne " jus " en arabe dialectal. L'amélioration de la qualité du Tassabount nécessite en préalable une bonne connaissance des étapes de préparation de ce produit (figure 1). L'analyse du procédé telle que nous l'avons faite révèle un mode de préparation assez simple qui comporte deux étapes principales : la préparation d'un extrait aqueux à base de plantes aromatiques et médicinales tout d'abord, puis l'obtention du jus.

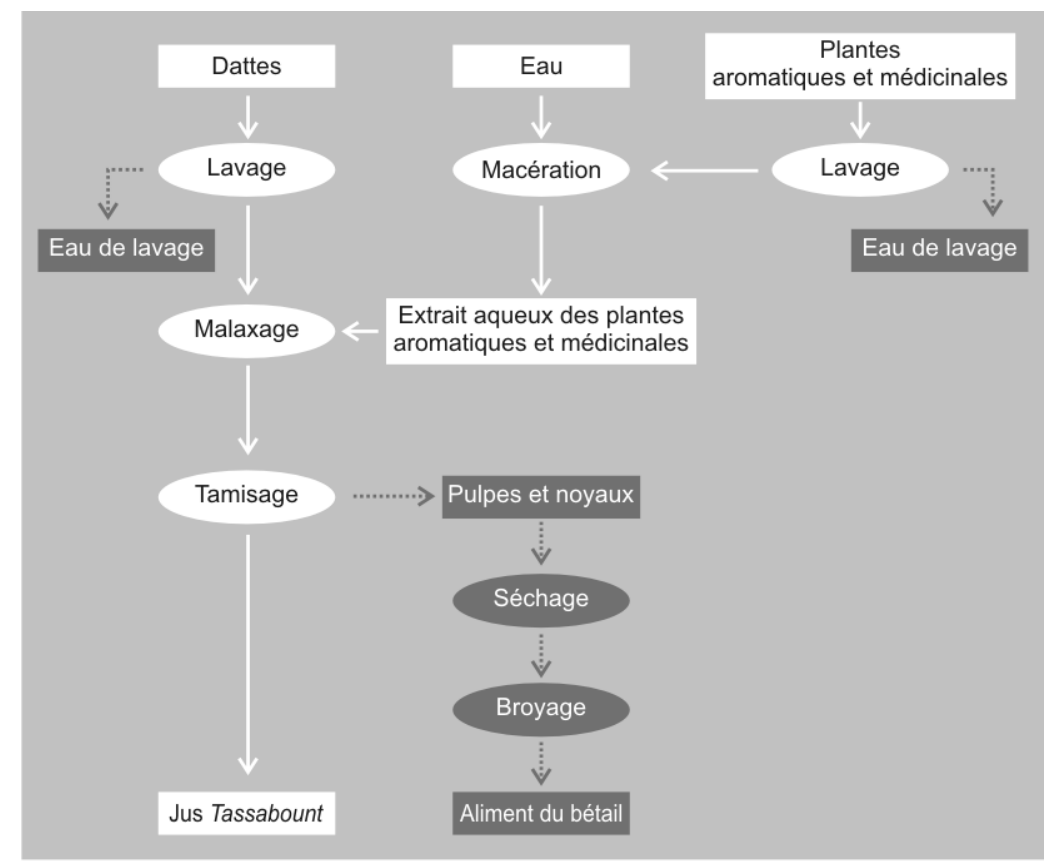

\subsubsection{Préparation d'un extrait aqueux à base de plantes aromatiques et médicinales}

L'extrait aromatique, fabriqué préalablement pour la préparation du Tassabount, est appelé localement Khmira, mot qui signifie "ferment ". Cette solution est traditionnellement préparée dans un pot de terre cuite ou dans une courge vidée et séchée. Cependant, de nos jours, certains ménages utilisent des bidons alimentaires en polychlorure de vinyle provenant généralement du recyclage de bidons de $5 \mathrm{~L}$ utilisés pour le conditionnement de l'huile de table. Le récipient est rempli d'eau dans laquelle macèrent, pendant deux à quatre jours, de petites quantités de diverses plantes aromatiques et médicinales préalablement triées, lavées et parfois regroupées en trois ou quatre bouquets et enveloppées dans un tissu. Selon la quantité d'eau, une à quatre dattes sont parfois ajoutées à ce mélange comme source supplémentaire d'hydrates de carbone. La préparation peut être par moment exposée au soleil afin d'accélérer la macération des plantes utilisées. La fermeture du récipient peut être hermétique ou perméable à l'air lorsque le pot est couvert avec un tissu propre. Le liquide jaune clair obtenu
Figure 1.

Étapes du procédé familial de préparation traditionnelle du jus de dattes Tassabount par les femmes oasiennes (Maroc). 
est riche en arômes et a un goût légèrement acide.

Notre enquête dans les oasis a révélé qu'environ trente plantes aromatiques et médicinales entraient dans la préparation du Tassabount (tableau I), qui, par suite, présente des vertus thérapeutiques et des notes aromatiques diverses selon les plantes utilisées. Le choix et le nombre des plantes utilisées peuvent varier d'une palmeraie à l'autre et d'un foyer à l'autre en fonction du rang social des artisans, de la disponibilité des plantes et de la préférence des oasiens. L'analyse de la liste des plantes utilisées dans la préparation de Tassabount montrent qu'elles se répartissent dans quatre catégories d'usages différents :

- les plantes à boisson : citron, lime, etc. ; - les plantes à parfum : rose pâle, basilic, etc. ;

- les plantes médicinales à grandes cultures et spontanées : henné, mandragore, etc. ;

- les plantes à tisanes et infusettes : menthe pouliot, thym, etc.

Il convient de noter que certaines plantes telles que le harmel et la mandragore sont classées dans la catégorie des plantes toxiques, et nécessiteraient donc que la population soit informée du danger de toxicité lié à leur utilisation non contrôlée.

\subsubsection{Obtention du jus Tassabount}

Après lavage, les dattes entières sont énergiquement pétries à la main dans une poterie (Gasâa ou Gasria) jusqu'à obtention d'une pâte homogène de couleur claire. À ce stade, l'extrait de plantes aromatiques et médicinales préalablement préparé est progressivement ajouté en malaxant la pâte jusqu'à obtention d'un mélange relativement visqueux. Au cours de cette étape, il se forme une mousse abondante rappelant celle du savon. Depuis peu, au lieu d'un pétrissage à la main, certains ménages utilisent un mixeur électrique qui facilite le broyage de la pulpe de dattes.

Le mélange obtenu après malaxage est finement tamisé pour écarter le retentât constitué des noyaux et d'une partie des pulpes, et récupérer le jus pulpeux (Tassabount). Après séchage et broyage, ce retentât, appelé localement Khamel, peut servir d'aliment au bétail. Cependant, dans certains cas, avant qu'il ne soit donné aux animaux, le retentât est lavé pour récupérer le maximum de ses substances solubles, dont les sucres et les composés d'arôme, et l'eau de lavage est ajoutée ensuite au jus pulpeux. Lors de cette préparation, il est primordial de travailler dans de bonnes conditions sanitaires afin d'obtenir un jus Tassabount de bonne qualité.

La préparation familiale du Tassabount est en étroite relation avec la richesse de la palmeraie en variétés et khalts (hybrides non sélectionnés issus de semis naturels, auxquels aucun nom n'a été attribué). Le choix des dattes utilisées dans la préparation de Tassabount est basé principalement sur trois critères (tableau II) :

- L'aptitude de la variété à la préparation de Tassabount : les dattes doivent avoir une consistance molle pour faciliter leur pétrissage et elles doivent être aptes à la formation d'une mousse blanche et abondante. Ce caractère mousseux du Tassabount est très recherché car, en plus de l'appréciation organoleptique, il revêt une importance spirituelle chez les oasiens pour lesquels il constitue un porte-bonheur.

- Les dattes doivent avoir de bonnes caractéristiques organoleptiques qui sont transmises au jus.

- La variété doit être disponible tout au long de l'année.

Il est également possible d'utiliser un mélange de variétés. Cependant, des variétés de consistance sèche à demi-sèche comme Jihel, Bouskri et Bourhare ne peuvent pas convenir à cette préparation.

\subsection{Propositions de développement d'un procédé standardisé pour la préparation du Tassabount}

L'exploitation commerciale du jus de dattes Tassabount sur le marché impose une standardisation du procédé familial et l'apport de savoir-faire complémentaires dans les domaines de la stabilisation du produit, de son conditionnement, ainsi que dans l'amélioration de la qualité globale de ce jus. De ce fait, l'amélioration du procédé de 
Tableau I.

Plantes aromatiques et médicinales utilisées traditionnellement par les femmes oasiennes dans la préparation du jus de dattes Tassabount au Maroc.

\begin{tabular}{|c|c|c|}
\hline Plantes & Nom vernaculaire & Nom scientifique d'après [4] \\
\hline Amandier amer & Louz Imor & Prunus amygdalus Stokes \\
\hline Armoise blanche & Shih & Artemisia herba-alba Asso. \\
\hline Basilic & Lahbaq & Ocimum basilicum L. \\
\hline Citronnier (fruit : citron) & Hamedh & Citrus limon (L.) Burm. \\
\hline Fumeterre & Oum Ibina & $\begin{array}{c}\text { Fumaria capreolata L. } \\
\text { F. officinalis L. } \\
\text { F. agraria Lag. } \\
\text { F. parviflora Lam. } \\
\text { Euphorbia obtusifolia Poiret } \\
\text { E. helioscopa L. }\end{array}$ \\
\hline Gaillonia & Sedra bayda & Gaillonia reboudiana Coss. et Dur. \\
\hline Girofle & Kerounfel, Ud annawar & $\begin{array}{l}\text { Eugenia cariophyllata Thunb. } \\
\text { [Syzygium aromaticum (L.) Merr.] }\end{array}$ \\
\hline Haloxylon & Erramt & Haloxylon scoparium Pomel \\
\hline Harmel (racine) & Al harmal & Peganum harmala L. \\
\hline Henné (feuilles) & Al henna & $\begin{array}{l}\text { Lawsonia inermis L. } \\
\quad \text { (L. alba Lamk.) }\end{array}$ \\
\hline Iris (racine) & Al âmbar & $\begin{array}{l}\text { Iris germanica } \mathrm{L} . \\
\text { I. florentina } \mathrm{L} \text {. }\end{array}$ \\
\hline Limettier (fruit : lime) & Lime & $\begin{array}{l}\text { Citrus limon (L.) Burm. } \\
\text { C. aurantiifolia Swingle }\end{array}$ \\
\hline Mandragore & Taryala & Mandragora autumnalis L. \\
\hline Menthe pouliot & Fliyû & Mentha pulegium L. \\
\hline Menthe ronde & Timijja & $\begin{array}{c}\text { M. suaveolens Ehr. } \\
\text { [M. rotundifolia (L.) Hudson] }\end{array}$ \\
\hline Muscade & Gouza tib, Bsibisa & Myristica fragrans Houtt \\
\hline Myrte & Errayhane & Myrtus communis L. \\
\hline Origan & Zartar & $\begin{array}{c}\text { Origanum compactum Benth. } \\
\text { O. vulgare L. }\end{array}$ \\
\hline Ormenis & Izri, Gartoufa & $\begin{array}{l}\text { Ormenis africana Jord. et Fourr. } \\
\text { O. scariosa (Ball.) Lit. et Maire }\end{array}$ \\
\hline Palmier dattier (fruit : datte) & Tmar & Phœnix dactylifera L. \\
\hline Renoncule & Oedn hallouf & Pulicaria arabica (L.) Cass. \\
\hline Romarin & Azir & Rosmarinus officinalis L. \\
\hline Rose pale & Ouard, Al ward al baldi & $\begin{array}{l}\text { Rosa damascena Mill. } \\
\text { R. centifolia Mill. }\end{array}$ \\
\hline Sarghine (racine) & Sarghina & Corrigiola telephiifolia Pour. \\
\hline Souchet rond & Tara, Tagha & Cyperus rotondus L. \\
\hline Thym & Azkeni, Azzîtra & $\begin{array}{c}\text { Thymus satureioides Coss. et Ball. } \\
\text { T. broussonetii Boiss. } \\
\text { T. pallidus Coss. } \\
\text { T. maroccanus Ball. } \\
\text { T. vulgaris }\end{array}$ \\
\hline Zygophylle (racine) & Al âggaya & $\begin{array}{c}\text { Gactulum album } \\
\text { Zygophyllum gaetulum Emb. et Maire } \\
\text { Z. waterloti Maire } \\
\text { Z. fontanesi Webb. }\end{array}$ \\
\hline
\end{tabular}


Tableau II.

Critères de choix et degrés d'appréciation des dattes de quelques variétés et khalts, utilisées dans la préparation du jus Tassabount selon les femmes oasiennes (Maroc).

\begin{tabular}{|c|c|c|}
\hline Variétés et khalts & Critères de choix & Degré d'appréciation \\
\hline Boufeggous & $\begin{array}{c}\text { Formation d'une pâte blanche } \\
\text { Formation d'une mousse blanche } \\
\text { Jus gras }\end{array}$ & Excellente $^{1}$ \\
\hline Bousthammi noire & $\begin{array}{c}\text { Formation d'une pâte blanche } \\
\text { Formation d'une mousse blanche } \\
\text { Très bon goût } \\
\text { Disponible toute l'année }\end{array}$ & Excellente \\
\hline Oum-Tassabount & $\begin{array}{l}\text { Datte molle } \\
\text { Bonne aptitude à la préparation de Tassabount }\end{array}$ & Excellente \\
\hline Aguellid & $\begin{array}{l}\text { Formation d'une pâte blanche } \\
\text { Formation d'une mousse blanche } \\
\text { Disponible toute l'année }\end{array}$ & Très bonne \\
\hline Oum-N'hal & Obtention d'un jus blanc & Très bonne ${ }^{2}$ \\
\hline Mah El-Baid & Datte molle & Très bonne \\
\hline Kounst (Kounsa) & Formation d'une mousse blanche & Bonne \\
\hline Tamellalt (Mellala) & $\begin{array}{l}\text { Datte mielleuse } \\
\text { Formation d'une mousse blanche }\end{array}$ & Bonne \\
\hline Khalts jaunes mielleux & $\begin{array}{l}\text { Datte molle mielleuse } \\
\text { Datte de couleur jaune }\end{array}$ & Bonne \\
\hline Iklane & Vertu médicinale & Passable \\
\hline Aceddam & Formation d'une mousse blanche & Passable \\
\hline
\end{tabular}

préparation familial pourrait se faire en intervenant sur les étapes de fabrication usuelle ; cela permettrait d'obtenir un jus artisanal amélioré et, éventuellement, d'introduire de nouvelles opérations aboutissant à la stabilisation du jus facilitant ainsi sa commercialisation. Les interventions pourraient se faire à différentes étapes de la préparation du jus : phases de préparation de l'extrait aromatique, du malaxage, de la stabilisation du produit et de son conditionnement en particulier.

\subsubsection{Préparation de l'extrait aromatique}

L'étape de macération des plantes aromatiques et médicinales se déroule typiquement dans des conditions non contrôlées (durée, température, etc.) et cela pourrait ne pas garantir l'innocuité de l'extrait. Une première action visant l'amélioration de la qua- lité du Tassabount consisterait donc à rechercher un procédé standard où les différentes étapes de préparation de cet extrait se feraient de manière reproductible. La nature, la disponibilité et la quantité de plantes aromatiques et médicinales choisies pourraient être ainsi définies de manière standard, ainsi que les conditions (durée et température) de macération. La préparation de cet extrait aromatique pourrait avoir une incidence sur ses qualités organoleptique et hygiénique et sur celles du jus qui en découlera. Il conviendra de l'étudier. À défaut d'un traitement ultérieur de stabilisation du jus, cette étape de "fermentation ", aboutissant à un extrait aromatique aqueux légèrement acide ( $\mathrm{pH}$ de l'ordre de 5) et riche en substances volatiles (composés d'arôme) pouvant avoir une activité antiseptique, pourrait inhiber la croissance des bactéries pathogènes et de la flore fongique. Cependant, il existe des possibilités de contamination 
importante lors des autres étapes de préparation du Tassabount, notamment celles du malaxage et du tamisage. Ces contaminations peuvent avoir en partie, comme origine, les conditions sanitaires inappropriées.

\subsection{2. Étape du malaxage}

L'étape du malaxage est celle du mélange de l'extrait aromatique aux dattes. À ce stade, le ratio en poids [extrait aromatique : datte] conditionne la qualité physico-chimique du jus Tassabount (Brix, solides insolubles en suspension, etc.) qui sera obtenu. Le choix de ce rapport devra être basé sur les caractéristiques du jus produit au niveau des oasis.

Les dattes utilisées dans la préparation du Tassabount sont récoltées, ramassées et conservées dans des conditions hygiéniques variables. À cet effet, les dattes peuvent subir, avant malaxage, un blanchiment visant, outre la destruction de certains germes et l'inactivation enzymatique, un attendrissement du fruit apte à faciliter les opérations de malaxage et de dénoyautage.

\subsection{3. Étape de la stabilisation du produit}

Au niveau des oasis, le jus Tassabount est consommé à l'état frais. Un traitement de stabilisation devrait permettre d'augmenter sa durée de vie en limitant les activités enzymatiques et microbiennes, sans pour autant affecter la qualité organoleptique de ce jus. Cette stabilisation pourrait se faire par ajout d'huiles essentielles provenant des plantes aromatiques et médicinales utilisées ou par application d'un traitement physique, comme la pasteurisation, en adoptant un barème adéquat de traitement (temps et température).

\subsubsection{Conditionnement}

Après stabilisation du jus, il conviendra d'adopter un conditionnement adéquat. Les techniques d'emballage et d'étiquetage devront être pratiquées dans de bonnes conditions hygiéniques et adaptées à un marché de produit de terroir. Pour cela, la labellisation d'origine de ce jus en tant que produit de la Réserve de Biosphère des Oasis du Sud Marocain (label environnemental mondial) pourrait être envisagée. Elle serait une traduction de la synergie entre la qualité d'un espace naturel et la qualité d'un produit qui en est issu, ce qui permettrait une valorisation supplémentaire et un désenclavement de la production oasienne face à un marché mondialisé.

\section{Conclusion}

L'étude que nous avons réalisée en milieu oasien a permis de mettre en évidence une pratique ingénieuse qui témoigne de l'effort réalisé depuis fort longtemps par les femmes de cet environnement afin de valoriser une partie, même minime, de leur production ou du moins de satisfaire leurs besoins en produits diversifiés à base de dattes.

Par ailleurs, nos travaux ont conduit à reconnaître le jus de dattes Tassabount comme un produit de terroir prometteur en misant sur ses qualités organoleptiques et ses vertus thérapeutiques; il conviendra de promouvoir ces atouts dans des programmes de valorisation technologique. Dans ce contexte, l'analyse préliminaire des pratiques familiales de préparation du Tassabount que nous avons réalisée a induit une série de réflexions sur les améliorations qui pourraient être entreprises à l'échelle expérimentale au niveau des différentes étapes de préparation du produit : préparation de l'extrait aqueux aromatique, malaxage, stabilisation et conditionnement du jus obtenu. L'amélioration de ces différentes étapes fera l'objet de travaux ultérieurs visant la mise au point d'un procédé amélioré de préparation de Tassabount ; conjointement, l'évaluation des qualités organoleptique, hygiénique et marchande du produit obtenu pourrait permettre d'envisager la transmission de ce procédé amélioré aux petites ou moyennes entreprises artisanales et/ou industrielles.

À terme, la préservation et la valorisation de ce savoir-faire indigène de préparation du jus de dattes permettront aux consommateurs de revenir au label naturel des produits de terroirs et de renouer avec une alimentation traditionnelle, localement génératrice de valeur ajoutée et considérée comme plus goûteuse et naturelle. 


\section{Remerciements}

Nous remercions les femmes oasiennes et toutes les personnes qui ont contribué efficacement à la réalisation de cette étude sur le terrain. Nous remercions également :

- le projet RAB98G31 (IPGRI/PNUD/ FEM/INRA) intitulé "Gestion participative des ressources génétiques du palmier dattier dans les oasis du Maghreb ", qui est un projet maghrébin englobant le Maroc, l'Algérie et la Tunisie,

- le programme de Recherche Agronomique pour le Développement PRAD 05/03 intitulé "Transformation des dattes de faible valeur marchande en jus : évaluation de la qualité et de la stabilité au stockage ", qui est un programme de coopération établi entre l'INRA (Maroc) et le CIRAD (France).

\section{Références}

[1] Harrak H., Chetto A., Valorisation et commercialisation des dattes au Maroc, INRA Ed., Marrakech, Maroc, 2001, 222 p.

[2] Zirari A., Harrak H., Chetto A., Alaoui Rachidi M., Outlioua K., Réhabilitation de la diversité génétique du palmier dattier dans la palmeraie de Fezouata, Projet RAB98G31, Gestion participative des ressources génétiques du palmier dattier dans les oasis du Maghreb, Rapp. Atelier Diagn. Particip., 21-26 avril 2003, IPGRI / INRA Maroc, Zagora, Maroc, 2003, 96 p.

[3] Zirari A., Chetto A., Harrak H., Alaoui Rachidi M., Outlioua K.,. Diagnostic participatif de la diversité génétique du palmier dattier et de son utilisation dans le Drâa: cas de l'oasis de Fezouata, in : Boulanouar B., Kradi C. (Éds.), Symp. Int. Dév. Agric. Durable Syst. Oasiens, INRA Éd., Rabat, Maroc, 2005, pp. 66-71.

[4] Sijelmassi A., Les plantes médicinales du Maroc, Le Fennec Éd., Casablanca, Maroc, 1996, 285 p.

\section{Hacia una valorización del buena gestión local de los oasis: el caso del jugo} de los dátiles Tassabount en Marruecos.

Resumen - Introducción. Los oasis marroquíes tienen la reputación por la buena gestión ingeniosa por sus habitantes en materia de gestión y de preservación de recursos naturales. El dátil, principal producto de los oasis, beneficia ampliamente de dicha ingeniosidad que conoce, hoy en día, una regresión y una falta de valorización. Nuestro estudio tuvo como objetivo el archivo y el análisis de la buena gestión de la transformación de dátiles en jugo, localmente denominado Tassabount, y proponer un modo de valorización. Material y métodos. La metodología empleada para el trabajo de archivo y de análisis de conocimientos locales de transformación de los dátiles en jugo se basó en un acercamiento participativo que implicaba un centenar de mujeres habitantes del oasis de valle del Drâa (Marruecos). Resultados y discusión. Nuestro estudio puso en evidencia una práctica ingeniosa en la transformación local de los dátiles en jugo. Dicha buena gestión, desarrollada por las mujeres habitantes del oasis está estrechamente relacionada con la diversidad genética de la palmera de dátil (variedades e híbridos). Además, el Tassabount se identifica como producto prometedor a valorizar, apostando por sus cualidades organolépticas y por sus virtudes terapéuticas. Por otro lado, la descripción y el análisis del procedimiento familiar de preparación del Tassabount permitieron identificar las vías posibles de mejora para su elaboración y su estabilización en vista de su traspaso a las empresas artesanales y/o industriales.

Marruecos / Pboenix dactylifera / dátil / plantas medicinales / hierbas culinarias / jugo de frutas / oasis / tecnología tradicional / modernización / tecnología apropiada / transferencia de tecnología 